\title{
Magnetic Fields in the Galactic Center
}

\author{
G. Novak \\ Department of Physics and Astronomy, Northwestern University, Evanston, IL, 60208, USA
}

\begin{abstract}
.
Within the central few hundred parsecs of the Galaxy we find a magnetic field with a rich and complex filamentary structure. In some ways this structure is reminiscent of what we see in the Solar magnetosphere, but the size scale is a million times larger. Advances in the technology for far-infrared/submillimeter polarimetry are enabling us to acquire new data on interstellar magnetic fields in the Galactic center. We will review recent results in this area and attempt a synthesis with information obtained from non-thermal radio filament morphology, radio Faraday rotation, and Zeeman splitting.
\end{abstract}

Keywords: interstellar magnetic fields; Galactic center PACS: $98.35 . \mathrm{Eg}$

\section{INTRODUCTION}

In this paper we review what is known about the interstellar magnetic field in the Galactic center region, by which we mean the central $\sim 500$ parsecs of the Galaxy. This region is densely populated with stars and gas clouds, containing by some estimates $\sim 5 \%$ of the Galaxy's total stellar mass, and also $\sim 5 \%$ of its interstellar gas [1]. Within the Galactic center region, the density of stars is sharply peaked at the dynamical center itself, at the position of the compact non-thermal source Sagittarius $A^{*}[2,3,4]$ that marks the location of a black hole with a mass of 3-4 million solar masses [5, 6]. For Galactocentric radii greater than a few parsecs, however, it is the stars that make the dominant contribution to the gravitational potential, not the black hole. The same can be said for luminosity. Sgr A* is not a very luminous source, though it may have been brighter at some time in the past.

In comparison with the Galactic disk environment, the Galactic center is extreme in many ways. It contains, for example, unusual star clusters having extremely massive stars; hotter and denser molecular clouds that are also more turbulent; and brighter diffuse X-ray emission [1, 7]. Most of the interstellar gas in the Galactic center is in molecular clouds, and these are mostly confined to within $20 \mathrm{pc}$ of the Galactic plane.

\section{NON-THERMAL FILAMENTS}

Figure 1 shows non-thermal radio emission from the Galactic center. Besides the nonthermal shell source Sgr A(East) that is probably a supernova remnant, we see in this image a number of long filaments that are quite straight or have only gentle curvature. These structures are not seen elsewhere in the Galaxy, and are reminiscent of features seen in the Solar atmosphere. 


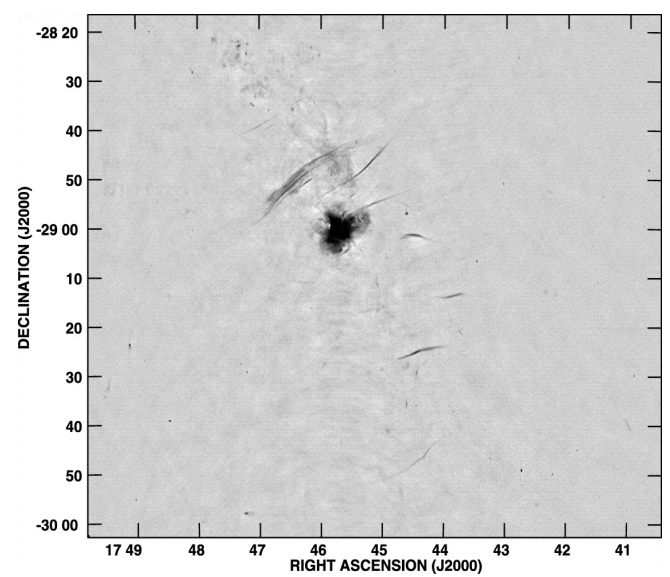

FIGURE 1. $90 \mathrm{~cm}$ continuum radiograph of the central $\sim 300 \mathrm{pc}$, showing the brightest of the Galactic center non-thermal filaments (NTFs) [8]. The Galactic plane runs from 11 o'clock to five o'clock, with Galactic North at upper right and Galactic East at upper left. The brightest feature in this image is the non-thermal shell source Sagittarius A(East), thought to be a supernova remnant. The location of Sgr A* (in projection, at least) lies inside Sgr A(East) but near its Western edge. The NTFs run more or less perpendicular to the Galactic plane, especially where they cross it. Note the bright bundle of NTFs that is displaced toward the Galactic East direction with respect to Sgr A(East). This group of NTFs is referred to as the Radio Arc.

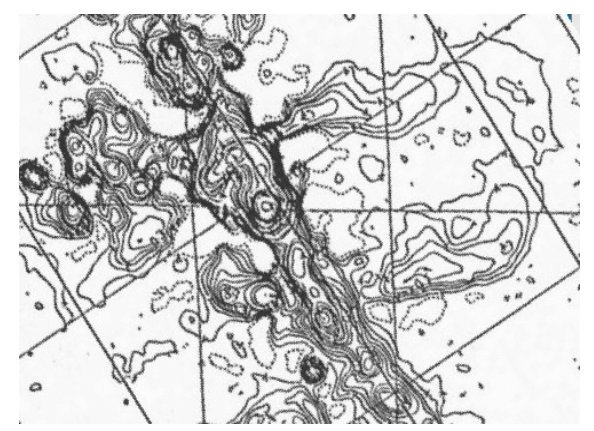

FIGURE 2. $3.5 \mathrm{~cm}$ radio continuum image of the same region shown in Figure 1 [9]. This single-dish image has been filtered to remove the very lowest spatial frequencies. The bright point-like peak near the center is Sgr A(East). Two lobes can be seen extending toward positive Galactic latitudes. These are referred to as the Eastern and Western lobes and and they appear to form a continuous structure that may be tracing an outflow. Note also that the Radio Arc of Figure 1 can also be seen in this image, and here we can see that it has extensions toward Galactic North and Galactic South. These are referred to as the Northern and Southern plumes, respectively. Thus, the Northern plume is more or less the same thing as the Eastern lobe.

Since their discovery about two decades ago [10] we have learned that these nonthermal filaments (NTFs) are highly polarized, with polarization E-vector orthogonal to the filament [11]. Thus they are synchrotron emission structures and they trace out lines of magnetic force. Their overall geometry suggests a large scale poloidal 


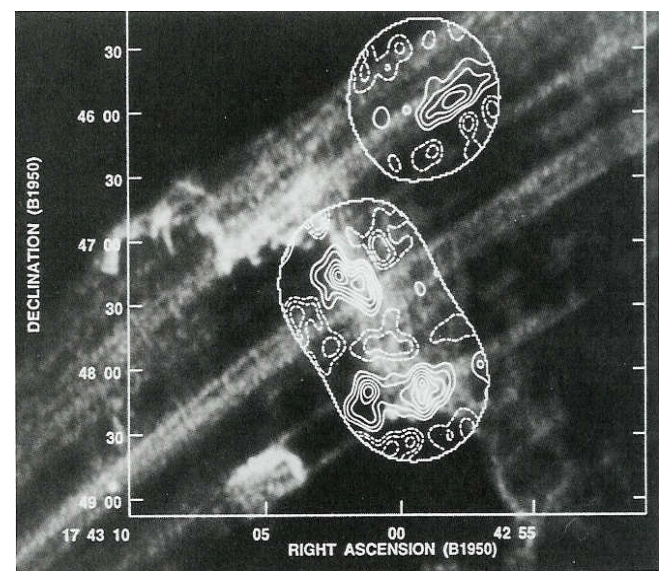

FIGURE 3. Close-up view of the spot where the Radio Arc NTF bundle crosses the Galactic plane, with gray-scale showing $20 \mathrm{~cm}$ continuum emission and contours showing CS line emission [12]. The fleecy structure that extends parallel to the Galactic plane and crosses the NTFs is the thermal radio source known as the "Sickle". The $20 \mathrm{~cm}$ image (originally from reference [13]) represents evidence for interaction between thermal and non-thermal sources: Specifically, note that some of the NTFs die out or get much fainter precisely where they cross the Sickle.

magnetic field filling the Galactic center region. In this interpretation, the filaments show where relativistic electrons (that have been injected by some unknown mechanism) are spiraling along field lines thus illuminating selected flux tubes within an ordered, poloidal large-scale field.

The image in Figure 1 was made with an interferometer, but the one in Figure 2 was made with a single dish radio telescope and thus shows extended emission. Because of the shorter wavelength used for this observation, this image shows both thermal (photoionized) and non-thermal (synchrotron) emission. Two lobes seen in this image appear to be part of a limb-brightened outflow-like structure extending toward Galactic North. These are referred to as the Galactic Center Radio Lobes (GCRL). The Radio Arc shows up in both Figure 1 and Figure 2. In the latter, its extensions (the "plumes") may be seen.

Figure 3 shows evidence for interaction between the NTFs of the Radio Arc and a thermal radio source that is associated with a molecular cloud seen here in CS line emission. The NTFs show little sign of bending or distortion at the site of interaction. Galactic center molecular clouds have complex kinematics, but one can use typical values for turbulent velocity and density to obtain a rough estimate of the ram pressure with which the cloud is impacting the NTFs. It has been argued [7] that since the field shows little bending it must have an energy density comparable to this ram pressure. This leads to a minimum strength of $1 \mathrm{mG}$ for the field in the filaments. Because it is difficult to confine such a strong field to a narrow filament, it has been further argued that there must be a pervasive poloidal field with a strength of $>1 \mathrm{mG}$ filling the Galactic center region [7]. This hypothesized strong ordered field would dominate the energetics of the ISM.

The acceleration mechanism for the relativistic electrons that light up the NTFs has 


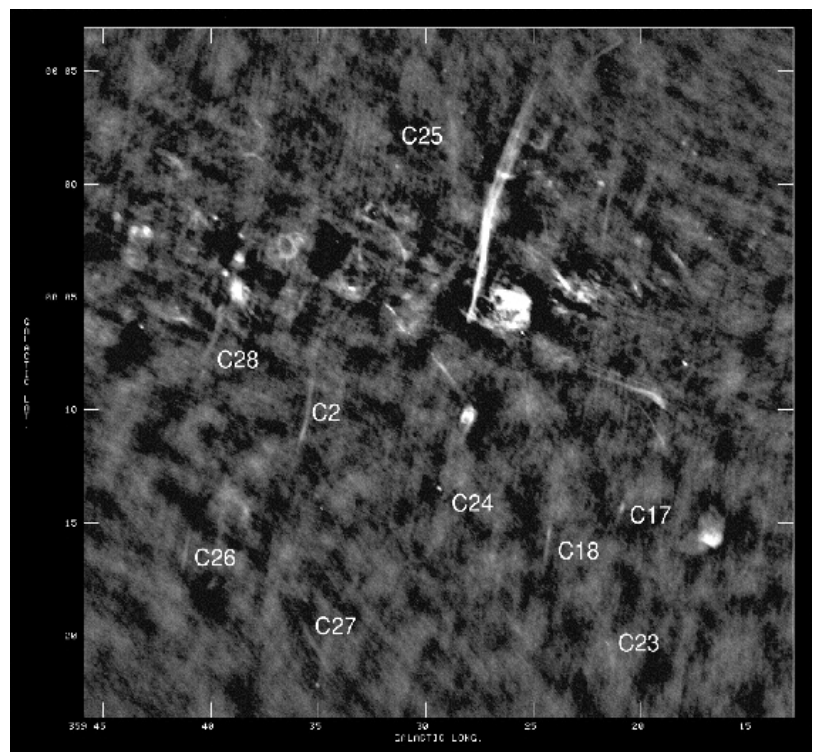

FIGURE 4. Close-up view of one of the bright NTFs, with Galactic plane running horizontally this time [19]. The image shows several examples of the numerous fainter NTFs that have been discovered only very recently $[19,8]$. These new faint NTFs are shorter and their orientations are varied, showing no tendency to be perpendicular to the Galactic plane. The bright NTF in this image can be seen in Figure 1 at declination $-29^{\circ} 25^{\prime}$.

been explored via numerous models (see reference [14] for a recent review). Here we will not attempt to review all models nor even all classes of models, but we will note that two energy sources that are commonly invoked are winds and shocks associated with star formation $[15,16]$ and the motion of molecular clouds in the gravitational potential of the Galactic center $[17,12,18]$. Many models rely on an instability to form the NTF. This involves field compression and thus an increase in field strength within the filament. Thus, even if the large-scale poloidal field in the Galactic center were much weaker than $1 \mathrm{mG}$, it might still be possible to create filaments having $\sim \mathrm{mG}$ fields, via such instability mechanisms.

An exciting very recent development that will have a large impact on our understanding of the NTFs and the Galactic center magnetic field is the discovery of numerous new fainter NTFs $[8,19]$. As can be seen in Figure 4, the faint NTFs are also shorter. Interestingly, they show no tendency to be perpendicular to the Galactic plane, but instead show no preferred orientation. Like the brighter NTFs of Figure 1, the fainter NTFs are generally confined to Galactic longitudes lying between the two lobes of Figure 2. These new fainter NTFs are believed to represent the same basic phenomenon as the brighter NTFs [8, 19].

The new faint NTFs reveal significant disorder in the Galactic center magnetic field, which is inconsistent with the "energetically dominant field" hypothesis discussed above. One might even argue that the NTF picture now seems more consistent with 


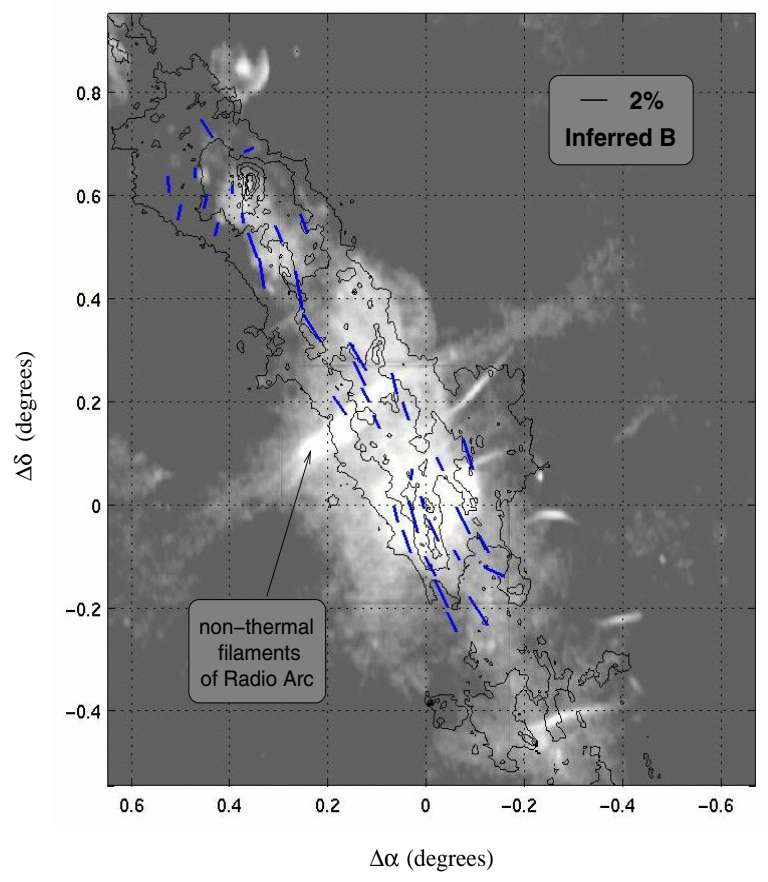

FIGURE 5. Submillimeter polarimetry of the Galactic center, shown with bars indicating magnetic field direction, and superposed on $90 \mathrm{~cm}$ radio continuum image [20]. Contours show the intensity of the submillimeter emission [21]. Note that the submillimeter polarimetry reveals a magnetic field running mostly parallel to the Galactic plane, indicating a globally toroidal field. The radio image is from reference [22].

a highly disordered field than with a globally poloidal field. In this "highly disordered field" hypothesis, the net magnetic flux crossing the Galactic plane would be insignificant. E.g., if the field in one bright NTF were to point toward Galactic North, then the field in another bright NTF might point toward Galactic South.

There is one class of models for the NTFs that fits nicely with the idea of a weak and disordered field. In these models the filaments are thought of as magnetized wakes similar to the tails of comets [23, 24]. The direction of the filaments is not determined by the direction of a pre-existing magnetic field but rather by that of an ionized wind that blows past a cloud.

These models have the advantage of explaining straight filaments without resorting to a strong and uniform magnetic field. However, it is difficult to imagine how they could apply to the Radio Arc filaments. For example, consider the gray-scale radio image shown in Figure 5. Note the Radio Arc and its extensions, the Northern Plume and Southern Plume. One would need to invoke a wind blowing from the far tip of one plume directly toward the Galactic plane, and it is hard to see how the filamentary structure of the Radio Arc (Fig. 1) could be created in this way. 
Thus, the "highly disordered field" hypothesis may not be correct and the possible existence of a poloidal field in the Galactic center remains an open question. But we can say that an ordered poloidal magnetic field with a strength of $\sim 1 \mathrm{mG}$ seems unlikely.

\section{ZEEMAN MEASUREMENTS}

The magnetic field in the atomic and molecular gas clouds can be sampled via measurements of Zeeman splitting of emission and absorption lines that reveal the strength of the line-of-sight component of the magnetic field. It is difficult to obtain Zeeman measurements for the Galactic center due to the very large line widths. The few measurements that have been obtained generally give $1-2 \mathrm{mG}[25,26,27]$, but this does not represent evidence in favor of a pervasive $\sim \mathrm{mG}$ field because the regions sampled are dense $\left(\sim 10^{4} \mathrm{~cm}^{-3}\right)$ and very turbulent $\left(\sim 10 \mathrm{~km} \mathrm{~s}^{-1}\right)$. For these conditions, $1-2 \mathrm{mG}$ is close to equipartition. One attempt was made to sample the field in the more tenuous and extended component of the neutral gas and it yielded only upper limits at the 0.3 $\mathrm{mG}$ level [28].

\section{FARADAY ROTATION MEASUREMENTS}

There is an extended ionized component to the Galactic center gas that produces very strong Faraday rotation (up to $5000 \mathrm{rad} \mathrm{m}^{-2}$ ). Such high rotation measures are not seen elsewhere in the Galaxy, and this ionized gas has been referred to as the Galactic center Faraday screen. Background sources that have been used to probe this Faraday screen include the Northern and Southern Plumes [29] and the NTFs [30, 31]. By separately estimating the column density of the ionized gas and furthermore assuming a uniform density, several authors have inferred magnetic field strengths of order $\sim 10^{-2} \mathrm{mG}$ $[29,30]$. To the extent that the medium is clumped this method will underestimate the field. Nevertheless, these measurements seem inconsistent with the idea of a pervasive $\sim \mathrm{mG}$ magnetic field in the Galactic center.

\section{SUBMILLIMETER AND FAR-INFRARED POLARIMETRY}

Polarimetry of far-IR/submillimeter thermal emission from magnetically aligned dust grains provides a way to trace magnetic field lines, or rather their projection onto the plane of the sky. This method is weighted toward dust column density so favors the denser cooler molecular clouds. This technique has been applied to various clouds in the Galactic center [32, 33, 34, 35, 36, 37]. The large-scale configuration of the field in the Galactic center molecular gas is toroidal, as can be seen in the large-scale submillimeter polarization map shown in Figure 5. Here the submillimeter "B-vectors" are shown superposed on a radio continuum image of the bright NTFs that might or might not be tracing a globally poloidal field, as discussed above.

For sight-lines near the Radio Arc, the submillimeter B-vectors are orthogonal to the NTFs (Fig. 5). This is presumably a line-of-sight superposition of two separated regions 


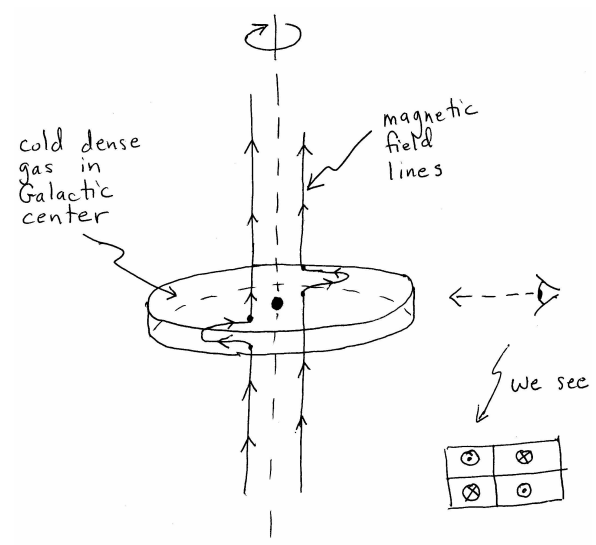

FIGURE 6. Cartoon showing how shearing of magnetic field lines within the differentially rotating layer of molecular gas at the Galactic center might deform a hypothetical globally axial field. Viewed from our vantage point in the Milky Way's disk, this deformation would produce a specific pattern in the line-of-sight component of the field. This is shown at lower right. Note that the direction of the line-ofsight component is opposite in adjacent quadrants, forming a pattern that is anti-symmetric with respect to both latitude and longitude. In the cartoon, the globally axial field is drawn pointing toward Galactic North. If it were to instead point toward Galactic South then all magnetic field directions should be reversed.

containing orthogonal fields. Even though the field in the molecular gas is predominantly toroidal, it is not surprising to find regions in the Galactic plane where the field runs perpendicular to the plane. This is because the molecular gas has very low filling factor [20].

One way to understand why the field in the molecular gas is toroidal is to consider the interplay between gravity and magnetism. The molecular gas has complex kinematics but is generally rotating in the same sense as Galactic rotation. The effect of the sharply peaked gravitational potential will be to strongly shear the gas and the field that is frozen into it. Unless magnetic forces are stronger than gravitational forces the magnetic field will not resist this shear and will end up with a toroidal configuration [32]. The critical field strength is $\sim 2 \mathrm{mG}$ [36]. From the good alignment of the magnetic field vectors with the Galactic plane (Fig. 5), we can conclude that the large scale field in the Galactic center must be well below $2 \mathrm{mG}$. This is barely consistent with a $\sim 1 \mathrm{mG}$ field.

Submillimeter polarimetric observations having higher resolution show specific regions where molecular cloud motions and/or winds appear to be bending the magnetic field, suggesting that magnetic field mapping may one day be useful for obtaining new information on the complex gas kinematics in the Galactic center [36, 37].

\section{SYNTHESIS: A TWISTED LARGE-SCALE FIELD?}

We will next consider the possibility that the large-scale poloidal field does exist, but we will assume that it has a strength well below $1 \mathrm{mG}$ and that it is confined to the volume 


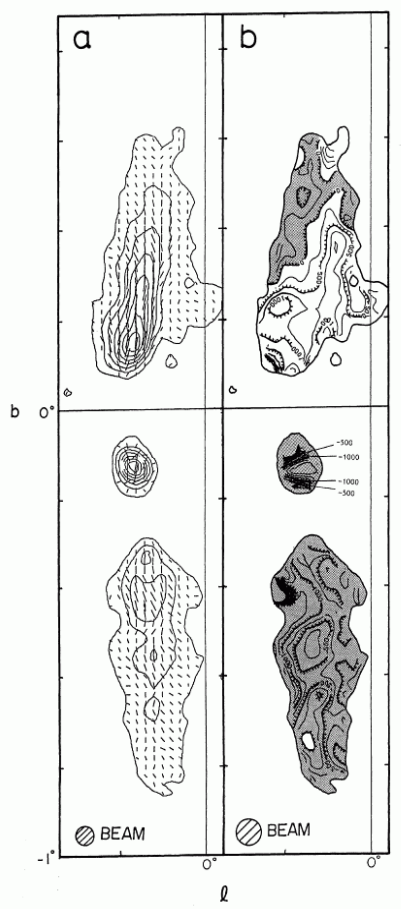

FIGURE 7. Faraday rotation measurements toward the synchrotron sources known as the Northern Plume and Southern Plume [29]. These plumes are described in the caption to Figure 2 and can also be seen in Figure 5 as the extensions of the Radio Arc toward positive and negative Galactic latitude, respectively. The left panel ("a") and right panel ("b") are both images of the same part of the sky. For both panels, Galactic longitude runs horizontally and Galactic latitude ranges from $-1.0^{\circ}$ to $+0.9^{\circ}$. The left panel shows the polarized intensity and inferred magnetic field angle for the two synchrotron-emitting plumes, corrected for Faraday rotation. The right panel shows the rotation measure. Rotation measures are negative below the plane and predominantly positive above the plane.

not occupied by molecular clouds. Recall that these molecular clouds are confined to a layer near the plane, with low filling factor. In this scenario, the field directions for all the bright filaments seen in Figure 5 are the same. I.e., either the fields inside these filaments all point toward Galactic North or they all point toward Galactic South.

Next consider the effect that the molecular clouds will have on this poloidal field. It seems very unlikely that the highly sheared molecular gas clouds, that are believed to be slowly drifting inwards [38], could move through the region containing the poloidal field without strongly affecting this poloidal field. The cartoon shown in Figure 6 shows how such a distortion might look, and how one could detect this distortion by observing the line-of-sight component of the field. Since the molecular gas has low filling factor, there would likely be places where the field is not distorted at all (see previous section), but on the largest scales one might expect to be able to observe the pattern shown in the lower right of Figure 6, or its inverse corresponding to the case where the global field is 


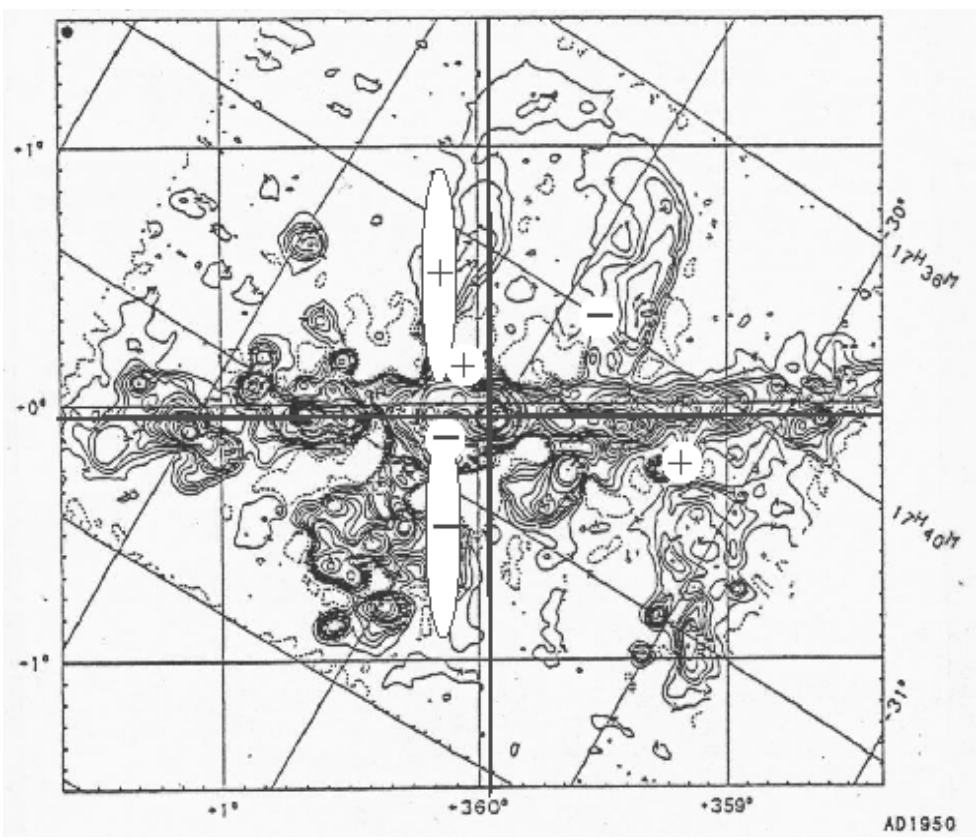

FIGURE 8. Illustration showing the sign of the Faraday Rotation measure for various Galactic center sources. The background image is the same as in Figure 2. The superposed oval-shaped regions indicate schematically the pattern seen in the Northern and Southern plumes (Fig. 7). The four superposed circles show results for NTFs lying within one degree of Sgr A*. (References are given in the text.) Positive Faraday rotation measure corresponds to field pointing toward the observer. Note the similarity of the pattern seen in this illustration to the pattern shown in Figure 6.

directed toward Galactic South (see caption to Fig. 6).

Faraday rotation provides a method to probe the line-of-sight field component and to thus search for the pattern shown in Figure 6 (see above section on "Faraday Rotation Measurements"). Indeed, a theoretical model for the Galactic center incorporating the idea of a magnetic twist like that shown in Figure 6 was published by Y. Uchida, Y. Sofue, and K. Shibata two decades ago [39, 40]. These authors pointed out that the field reversal that occurs between the Northern Plume and Southern Plume (shown in Fig. 7) is consistent with the "magnetic twist" in their model. The purpose of their model was to explain the GCRL structure seen in Figure 2 as a magnetically driven gas outflow.

Since the publication of the model of Y. Uchida and coworkers, four separate studies have been carried out in which the "Galactic center Faraday screen" (see above section on "Faraday Rotation Measurements") has been probed using NTFs lying within a degree of Sgr A* as background sources [41, 11, 30, 31]. It was recently pointed out [20] that if one considers the signs of the rotation measure for these new observations together with the signs of the rotation measure for the Northern and Southern plumes, then one finds agreement with the pattern expected for a magnetic twist. This is shown in Figure 8. If this agreement is not merely an accident resulting from small sample size, 
then the poloidal (or axial) field must point toward Galactic North. We note that for all four of the NTF Faraday rotation studies, the rotation measure is high. For three out of the four, there is no sign reversal across the measured area of the filament. The exception is the circle with negative rotation measure lying in the lower left quadrant of Figure 8, but even here one sign is clearly dominant: The rotation measure varies from $-5500 \mathrm{rad}$ $m^{-2}$ to $+350 \mathrm{rad} \mathrm{m}^{-2}$ [41].

We have suggested that patterns seen in Faraday rotation data provide a link between the toroidal field in the molecular gas and a possible poloidal (or axial) field as traced by the brighter NTFs. Note that the fields traced by the NTFs are permeating a gas where the temperature exceeds one million Kelvin, while the fields in the molecular clouds are permeating gas at $\mathrm{T} \sim 50-100 \mathrm{~K}$ and the fields sampled by Faraday rotation exist in a photo-ionized gas at an intermediate temperature of $\mathrm{T} \sim 10^{4} \mathrm{~K}$. From this point of view, it is natural to think that the Faraday data might provide a link between the field in the molecular clouds and the field traced by the NTFs. Although the Faraday data are too sparse to draw any firm conclusions, we believe that the similarity between the patterns in Figures 6 and 8 warrants new and more extensive Faraday rotation measurements toward the Galactic center.

\section{CONCLUSIONS}

We draw the following conclusions concerning the magnetic field in the Galactic center:

1. We rule out the "energetically dominant field" hypothesis, in which a poloidal magnetic field with a strength of $\sim 1 \mathrm{mG}$ is pervasive within the Galactic center.

2. In the molecular regions where most of the gas resides, the large-scale configuration of the magnetic field is toroidal.

3. It is possible that a weaker poloidal field having considerable disorder may exist in the Galactic center diffuse gas. If so, Faraday rotation measurements provide a way to study the connection between this poloidal field and the toroidal field in the molecular gas. An analysis of the very limited Faraday rotation data currently available suggests that a poloidal field does exist and that it points toward Galactic North.

\section{ACKNOWLEDGMENTS}

We acknowledge support from the National Science Foundation, via awards AST0243156 and OPP-0130389, and we thank David Chuss, Mark Morris, and Farhad Yusef-Zadeh for useful discussions.

\section{REFERENCES}

1. P. G. Mezger, W. J. Duschl, and R. Zylka, Astronomy and Astrophysics Review, 7, 289 (1996).

2. R. Genzel, R. Schödel, T. Ott, A. Eckart, T. Alexander, F. Lacombe, D. Rouan, and B. Aschenbach, Nature, 425, 934 (2003).

3. A. M. Ghez, S. A. Wright, K. Matthews, D. Thompson, D. Le Mignant, A. Tanner, S. D. Hornstein, M. Morris, E. E. Becklin, and B. T. Soifer, ApJ, 601, L159 (2004). 
4. F. K. Baganoff, M. W. Bautz, W. N. Brandt, G. Chartas, E. D. Feigelson, G. P. Garmire, Y. Maeda, M. Morris, G. R. Ricker, L. K. Townsley, and F. Walter, Nature, 413, 45 (2001).

5. A. M. Ghez, M. Morris, E. E. Becklin, A. Tanner, and T. Kremenek, Nature, 407, 349 (2000).

6. R. Schödel, T. Ott, R. Genzel, A. Eckart, N. Mouawad, and T. Alexander, ApJ, 596, 1015 (2003).

7. M. Morris, and E. Serabyn, ARA\&A, 34, 645 (1996).

8. T. N. LaRosa, M. E. Nord, T. Joseph, W. Lazio, and N. E. Kassim, ApJ, 607, 302 (2004).

9. R. F. Haynes, R. T. Stewart, A. D. Gray, W. Reich, P. Reich, and U. Mebold, A\&A, 264, 500 (1992).

10. F. Yusef-Zadeh, M. Morris, and D. Chance, Nature, 310, 557 (1984).

11. C. C. Lang, M. Morris, and L. Echevarria, ApJ, 526, 727 (1999).

12. E. Serabyn, and M. Morris, ApJ, 424, L91 (1994).

13. F. Yusef-Zadeh, and M. Morris, AJ, 94, 1178 (1987).

14. G. V. Bicknell, and J. Li, Publications of the Astronomical Society of Australia, 18, 431 (2001).

15. J. Nicholls, and E. T. Le Strange, ApJ, 443, 638 (1995).

16. R. Rosner, and G. Bodo, ApJ, 470, L49 (1996).

17. G. Benford, ApJ, 333, 735 (1988).

18. H. Lesch, and W. Reich, A\&A, 264, 493 (1992).

19. F. Yusef-Zadeh, J. W. Hewitt, and W. Cotton, ApJS, 155, 421 (2004).

20. G. Novak, D. T. Chuss, T. Renbarger, G. S. Griffin, M. G. Newcomb, J. B. Peterson, R. F. Loewenstein, D. Pernic, and J. L. Dotson, ApJ, 583, L83 (2003).

21. D. Pierce-Price, J. S. Richer, J. S. Greaves, W. S. Holland, T. Jenness, A. N. Lasenby, G. J. White, H. E. Matthews, D. Ward-Thompson, W. R. F. Dent, R. Zylka, P. Mezger, T. Hasegawa, T. Oka, A. Omont, and G. Gilmore, ApJ, 545, L121 (2000).

22. T. N. LaRosa, N. E. Kassim, T. J. W. Lazio, and S. D. Hyman, AJ, 119, 207 (2000).

23. S. N. Shore, and T. N. Larosa, ApJ, 521, 587 (1999).

24. R. B. Dahlburg, G. Einaudi, T. N. LaRosa, and S. N. Shore, ApJ, 568, 220 (2002).

25. N. E. B. Killeen, K. Y. Lo, and R. Crutcher, ApJ, 385, 585 (1992).

26. R. L. Plante, K. Y. Lo, and R. M. Crutcher, ApJ, 445, L113 (1995).

27. R. M. Crutcher, D. A. Roberts, D. M. Mehringer, and T. H. Troland, ApJ, 462, L79 (1996).

28. K. I. Uchida, and R. Guesten, $A \& A, 298,473$ (1995).

29. M. Tsuboi, M. Inoue, T. Handa, H. Tabara, T. Kato, Y. Sofue, and N. Kaifu, AJ, 92, 818 (1986).

30. A. D. Gray, J. Nicholls, R. D. Ekers, and L. E. Cram, ApJ, 448, 164 (1995).

31. F. Yusef-Zadeh, M. Wardle, and P. Parastaran, ApJ, 475, L119 (1997).

32. M. W. Werner, J. A. Davidson, M. Morris, G. Novak, S. R. Platt, and R. H. Hildebrand, ApJ, 333, 729 (1988).

33. R. H. Hildebrand, D. P. Gonatas, S. R. Platt, X. D. Wu, J. A. Davidson, M. W. Werner, G. Novak, and M. Morris, ApJ, 362, 114 (1990).

34. R. H. Hildebrand, J. A. Davidson, J. Dotson, D. F. Figer, G. Novak, S. R. Platt, and L. Tao, ApJ, 417, 565 (1993).

35. G. Novak, J. L. Dotson, C. D. Dowell, P. F. Goldsmith, R. H. Hildebrand, S. R. Platt, and D. A. Schleuning, ApJ, 487, 320 (1997).

36. G. Novak, J. L. Dotson, C. D. Dowell, R. H. Hildebrand, T. Renbarger, and D. A. Schleuning, ApJ, 529, 241 (2000).

37. D. T. Chuss, J. A. Davidson, J. L. Dotson, C. D. Dowell, R. H. Hildebrand, G. Novak, and J. E. Vaillancourt, ApJ, 599, 1116 (2003).

38. A. A. Stark, J. Bally, O. E. Gerhard, and J. Binney, MNRAS, 248, 14P (1991).

39. Y. Uchida, Y. Sofue, and K. Shibata, Nature, 317, 699 (1985).

40. K. Shibata, and Y. Uchida, Publications of the Astronomical Society of Japan, 39, 559 (1987).

41. F. Yusef-Zadeh, and M. Morris, ApJ, 322, 721 (1987). 